(b) When the inner cylinder is at rest and the outer cylinder is rotating with angular velocity $\omega_{2}$, the velocity $v_{\varphi}$ is given by

$$
v_{\varphi}=r_{2}^{\lambda+1} \omega_{2}\left(r_{1}^{2 \lambda}-r^{2 \lambda}\right) / r^{\lambda}\left(r_{1}^{2 \lambda}-r_{2}^{2 \lambda}\right) .
$$

The moment of the fluid $M_{2}$ on the outer cylinder is

$$
M_{2}=2 \pi r_{2}^{2} h \mu \omega_{2}\left[\frac{\lambda\left(r_{1}^{2 \lambda}+r_{2}^{2 \lambda}\right)}{\left(r_{2}^{2 \lambda}-r_{1}^{2 \lambda}\right)}-1\right],
$$

where $h$ is the length of the cylinder. Of course the equation determines $m / \nu$. If $M_{2}$ and $m$ are known, $\nu$ can be determined. (c) When a single cylinder rotates with angular velocity $\omega_{1}$, the velocity $v_{\varphi}$ and the moment $M_{1}$ are given, respectively, by

$$
\begin{gathered}
v_{\varphi}=r_{1}^{\lambda+1} \omega_{1} / r^{\lambda}, \\
M_{1}=-2 \pi r_{1}^{2} h \mu(\lambda+1) \omega_{1} .
\end{gathered}
$$

We note when $\lambda=1$, i.e., in the absence of the magnetic field, all the above equations tend to classical results.?

Currents and the electric fields. The induced electromotive forces and the current given by them are given by

$$
\begin{gathered}
\mathbf{E}=(\mathbf{v} \times \mathbf{B})=-\left(v_{\varphi} \bar{\mu} A / r\right) \hat{z}, \\
\mathbf{J}=\sigma \mathbf{E}=-\left(\sigma \bar{\mu} A v_{\varphi} / r\right) \hat{z},
\end{gathered}
$$

both acting parallel to the cylinder.

I thank Professor P. L. Bhatnagar for his guidance and help in the work.

${ }^{1}$ V. J. Rossow, N. A. C. A. Rept. No. 1358 (1958).

S. I. Pai, Viscous Flow Theory. I. Laminar Flow (D. Van Nostrand Company, Inc., Princeton, New Jersey, 1956).

\section{A Novel Device for Bursting Shock-Tube Diaphragms}

\section{A. Roshko and D. Baganoff \\ Guggenheim Aeronautical Laboratory, California Institute of Technology, Pasadena, California} (Received August 21, 1961)

$\mathrm{T}$ HE new GALCIT 17-in. low-pressure shock tube is equipped with a novel device which was designed to overcome some of the difficulties encountered in attempting to precisely select and control the bursting pressure of very thin diaphragms. This device is a cutter, shown in Figs. 1 and 2 , which consists of two blades set at right angles in a cruciform configuration and positioned in the tube on the low-pressure side of the diaphragm.

Under pressure, the diaphragm bulges out, as shown in the sequence of Fastax pictures in Fig. 3, and is neatly cut by the blades into four sections which open out as petals. The opening time of the
FIG. 1. Relation of cutter blades to diaphragm.

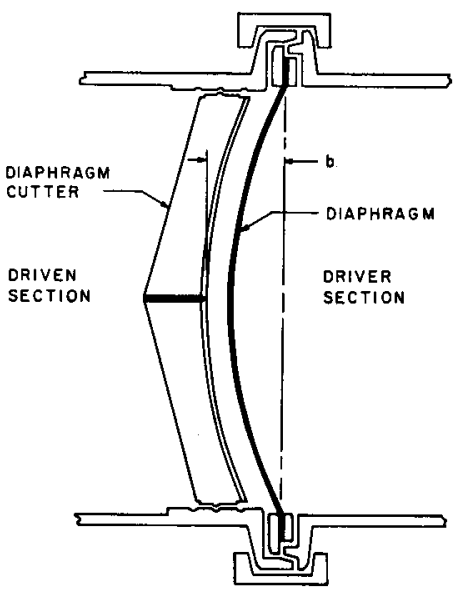

0.020-in. 1100-0 aluminum diaphragm, depicted in Fig. 3, is about 1 msec at a differential pressure of $55 \mathrm{lb} / \mathrm{in}^{2}$.

The bursting pressure is controlled by setting the distance $b$ between the blade and the diaphragm. In the shock tube installation shown in Fig. 2, the ends of the blades are attached to pads which ride in a spiral groove. Changes in blade position $b$ are made by rotating the cruciform in this thread; a pitch of 1 in. on the 17 -in. diameter allows precise positioning.

Calibrations of bursting pressure against blade setting are shown in Fig. 4. A given diaphragm can be burst at pressures in a range between a minimum and maximum value. The minimum value is the pressure required to cut the diaphragm with $b=0$. The maximum value is the pressure at which free bursting occurs, i.e., with the cutter removed; in this case the opening is ragged and pieces of the diaphragm may be torn off. With cutter installed, petaling is perfect, even for the points nearest the maximum bursting pressure. Straight and curved blades are used for small and large diaphragm deflections, respectively, as noted in Fig. 4. So long as first contact is at the center, the calibration appears to depend only on the blade setting $b$, but at the larger deflections the curved blade helps to ensure clean cutting out to the edges.

Fig. 2. Cutter installed in the GALCIT 17-in. low-pressure shock tube. The photograph shows the spiral groove by which the axial position of the diaphragm cutter is adjusted.

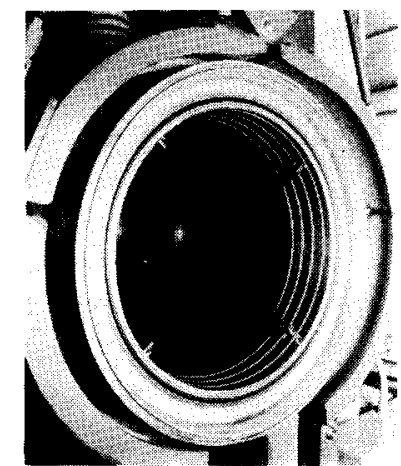




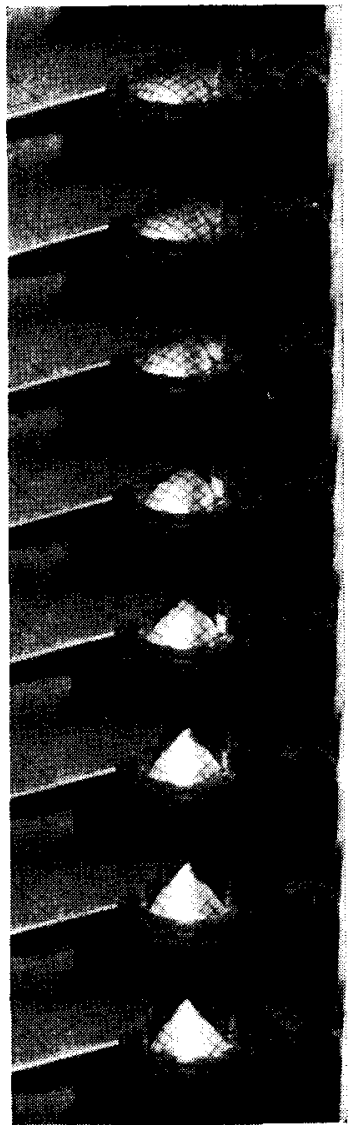

Fig. 3. Sequence showing diaphragm opening with a development model of the diaphragm cutter. The sequence of pictures was taken with a Fastax camera at a framing rate of 5600 frames/ sec. The first frame shows the inflated diaphragm touching the center portion of the curved blades of the cutter. The second frame shows the initiation of the opening process. The diaphragm was made of $1100-0$ aluminum 0.020 -in. thick and burst under a differential pressure of $55 \mathrm{lb} / \mathrm{in}^{2}$

The following features of this device are noteworthy:

(1) It gives precise control of the diaphragm opening pressure over a range of values for a given diaphragm. For very thin diaphragms, the range and degree of control is much better than with the scribing technique.

(2) It ensures leafing, thus reducing the probability of losing pieces of the diaphragm. Very thin scribed diaphragms (thickness/diameter $<0.001$ ) do not necessarily open along the scribe marks.

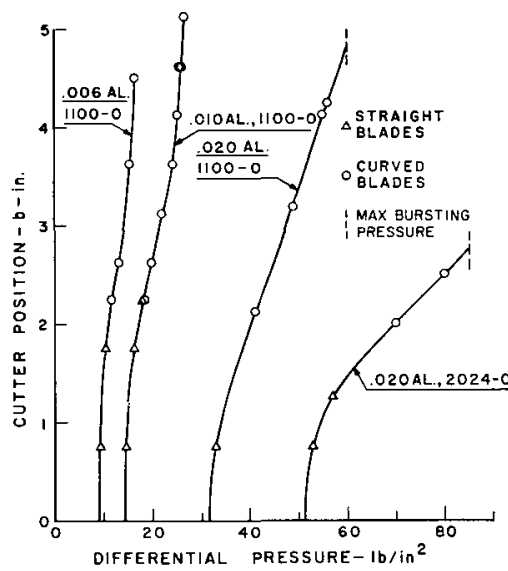

Fig. 4. Calibration curves. The cutter position is measured by the distance between the flat undeflected diaphragm and the center of the blades.
(3) The diaphragm mass and opening time are kept to a minimum. Because controlled opening is possible to nearly the free bursting pressure, the thinnest diaphragm may be used for given pressure; no extra thickness is needed for scribing.

(4) It is mechanically simple.

A further note should be added concerning items (2) and (3) listed above. The role of the diaphragm cutter is to initiate the diaphragm bursting, to direct the crack propagation, to provide a stress concentration for the traveling crack and to permit a diaphragm of minimum thickness to be used. The degree to which the diaphragm cutter has achieved these ends is vividly displayed by an unexpected problem which has arisen. The tip speed of the four petal ends of the diaphragm became great enough, on some runs, to spot-weld a one square $\mathrm{cm}$ area of each tip onto the stainless steel wall of the shock tube. For the 0.020-in. 1100-0 aluminum diaphragm, Fig. 4, this occurs for differential pressures of $40 \mathrm{lb} / \mathrm{in}^{2}$. and greater. This problem, at present, is being alleviated by the application of vacuum grease (an "antiflux") on a small area of the center of the diaphragm.

The work was supported by the National Aeronautics and Space Administration.

\section{Space-Charge Neutralized Ion Beams}

\author{
G. G. Kelley and O. B. Morgan \\ Oak Ridge National Laboratory* \\ Oak Ridge, Tennessee \\ (Received July 10, 1961)
}

$\mathrm{N}$ the course of experiments with intense hydrogen1 ion beams it has been found that these beams are automatically space-charge neutralized when focused by a magnetic solenoidal lens, unless an electron drain is provided. The effect of neutralization may be seen by visual observation. When the beam is neutralized it is narrow, sharply defined, and may be focused to an intense crossover. If, however, electrons are drawn out, for example by a positive bias on the target, a spreading may be seen which is consistent with the expected behavior of the beam with no neutralization.

A quantitative check was made at $50 \mathrm{kev}$ using a magnetic solenoidal lens of 6-in. aperture. An ion source was provided $4 \frac{3}{4}$ in. above the midplane of the lens. Below the midplane was a $1 \frac{1}{2}$-in. diameter limiting aperture at 18 in., a 1 -in. diameter aperture at 49 in., and a target at 50 in. A magnetic shield around the lens coil confined the field axially. Current to the target was measured calorimetrically. With this apparatus a 33-ma beam of $\mathrm{H}_{2}^{+}$ions was passed through the 1-in. diameter hole. This beam was observed to be less than 2 -in. diameter in the throat of the lens. It would be expected to be more 